\title{
Cytoskeletal and cellular adhesion proteins in zebrafish (Danio rerio) myogenesis
}

M.L. Costa, R. Escaleira,

M. Manasfi, L.F. de Souza and C.S. Mermelstein
Laboratório de Diferenciação Muscular e Citoesqueleto, Departamento de Histologia e Embriologia, Universidade Federal do Rio de Janeiro, Rio de Janeiro, RJ, Brasil

\section{Correspondence \\ M.L. Costa \\ Laboratório de Diferenciação \\ Muscular e Citoesqueleto \\ Departamento de Histologia e \\ Embriologia, UFRJ \\ 21949-590 Rio de Janeiro, RJ \\ Brasil \\ Fax: +55-21-2562-6480 \\ E-mail: manoelluiscosta@ufrj.br \\ Presented at SIMEC 2002 \\ (International Symposium \\ on Extracellular Matrix), \\ Angra dos Reis, RJ, Brazil, \\ October 7-10, 2002.}

Research supported by FAPERJ, FUJB-UFRJ and CNPq.

Received December 2, 2002

Accepted May 16, 2003

\begin{abstract}
The current myogenesis and myofibrillogenesis model has been based mostly on in vitro cell culture studies, and, to a lesser extent, on in situ studies in avian and mammalian embryos. While the more isolated artificial conditions of cells in culture permitted careful structural analysis, the actual in situ cellular structures have not been described in detail because the embryos are more difficult to section and manipulate. To overcome these difficulties, we used the optically clear and easy to handle embryos of the zebrafish Danio rerio. We monitored the expression of cytoskeletal and cell-adhesion proteins (actin, myosin, desmin, $\alpha$-actinin, troponin, titin, vimentin and vinculin) using immunofluorescence microscopy and video-enhanced, background-subtracted, differential interference contrast of 24- to 48-h zebrafish embryos. In the mature myotome, the mononucleated myoblasts displayed periodic striations for all sarcomeric proteins tested. The changes in desmin distribution from aggregates to perinuclear and striated forms, although following the same sequence, occurred much faster than in other models. All desmin-positive cells were also positive for myofibrillar proteins and striated, in contrast to that which occurs in cell cultures. Vimentin appeared to be striated in mature cells, while it is developmentally down-regulated in vitro. The whole connective tissue septum between the somites was positive for adhesion proteins such as vinculin, instead of the isolated adhesion plaques observed in cell cultures. The differences in the myogenesis of zebrafish in situ and in cell culture in vitro suggest that some of the previously observed structures and protein distributions in cultures could be methodological artifacts.
\end{abstract}

The main characteristic of muscle differentiation is the cytoskeletal rearrangement occurring to form the contractile apparatus, the myofibrils. This process involves the coordinated synthesis of several muscle-specific proteins and their assembly into almost crystalline structures. Since myofibrillogen-
Key words

- Cytoskeleton

- Cell adhesion

- Zebrafish

- Myogenesis esis involves redistribution of subcellular size structures, it has been followed more carefully in in vitro cell culture models (1). Some studies have been attempted on chick or mouse embryos, but in general these studies lack the refined level of detail that can be obtained in culture (2). To overcome these 
limitations, we have been studying myofibrillogenesis in zebrafish embryos using immunofluorescence against cytoskeletal proteins (3). We found some differences in zebrafish myofibrillogenesis compared to the established standard muscle formation sequence. The embryo muscles do not seem to form intermediate structures such as the stress fiber-like structure, and their myocytes striate and contract without fusion. We concluded that there are important allowances to be made before the in vitro-based myogenesis model in general and the myofibrillogenesis model in particular can be considered to correspond to the actual in vivo process. To further characterize the myofibril formation process, we have been studying the cytoskeletal attachments, anchorage regions that are essential for myogenesis and that should represent one of the major differ-

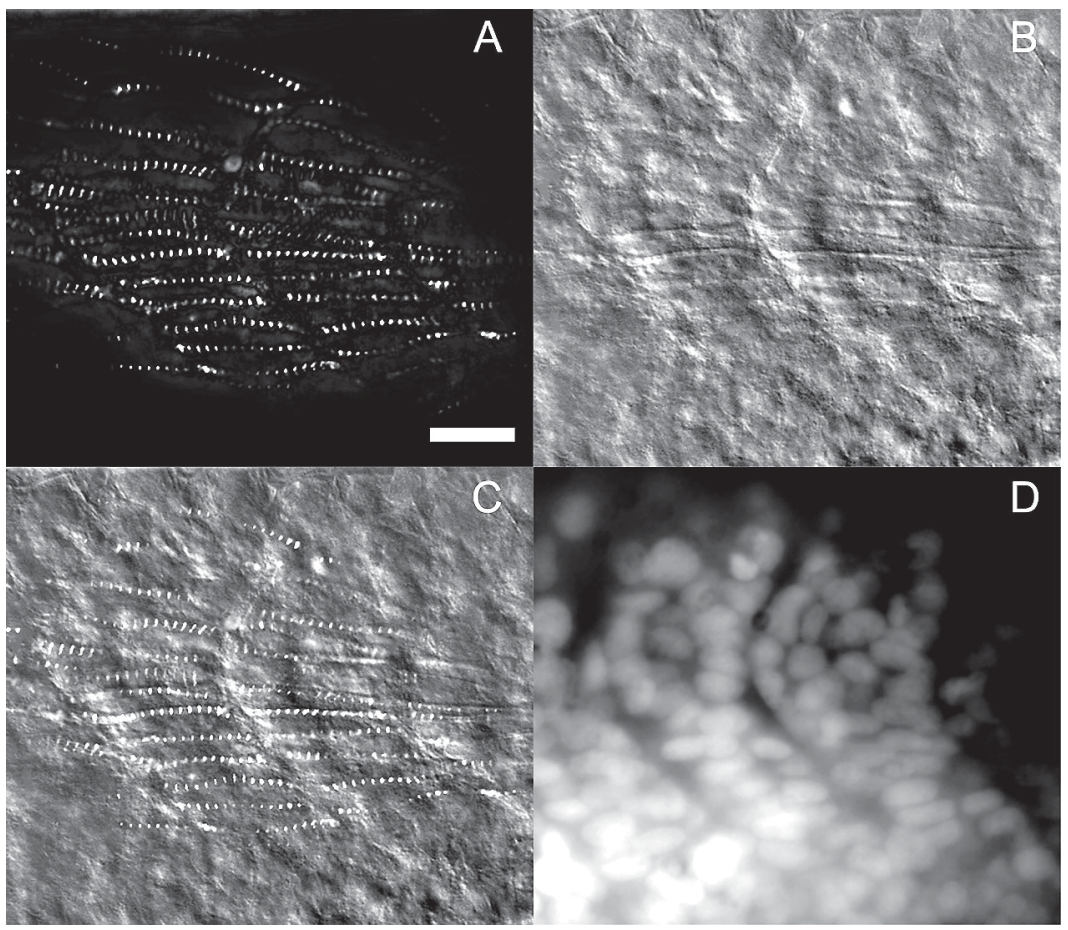

Figure 1. Precocious striations in young somites. Immunofluorescence stain of somites 2425 of 24-h zebrafish embryos using anti- $\alpha$-actinin antibodies (A) and video-enhanced, background-subtracted differential interference contrast (DIC) image (B). The perfect register of the striations with the DIC stain can be seen in $C$, where the two fields were superimposed. Several $\alpha$-actinin negative cells can be identified by their nuclear stain (4', 6-diamidino-2-phenylindole, D). The embryo head is on the right, the tail on the left. Scale bar: $10 \mu \mathrm{m}$. ences between the in vitro and in situ models.

The cytoskeleton is mechanically connected to the extracellular matrix through a series of proteins. In skeletal muscle, collagen fibers bind, through the adapter proteins fibronectin and laminin, to the transmembrane protein integrin. Integrin aggregates bind, through the adapter proteins vinculin, paxillin and talin, to actin microfilaments (4). During myogenesis, some cells attach to each other through their self-binding transmembrane cadherins, which in turn bind to catenins, which finally attach to actin microfilaments (5). Skeletal muscle has a third cytoskeletal attachment through the laminin receptors $\alpha$ - and $\beta$-dystroglycans, which bind to dystrophin, which then attaches to actin (6).

Most of those proteins have been shown to be involved in myogenesis, since their experimental or genetic disruption can lead to muscle defects. Thus, dystrophin has been identified as the missing gene in Duchenne muscular dystrophy (7). Antibodies to cadherin block myogenesis (8) and congenital muscle diseases have been related to mutations in integrin (9) and desmin (10) genes, among others.

The embryos were collected from our wild-type colony, and they were kept at $28^{\circ} \mathrm{C}$ under a controlled light cycle. With 24 to 48 $\mathrm{h}$ of development, they were fixed in $4 \%$ paraformaldehyde in PBS, permeabilized with $0.1 \%$ Triton X-100 in PBS, and stained with antibodies against $\alpha$-actinin (catalog number A-7811), vimentin (catalog number $\mathrm{V}-5255)$ and vinculin (catalog number V4505). All reagents were from Sigma (St. Louis, MO, USA), unless otherwise stated. They were stained with fluorescein isothiocyanate-conjugated secondary antibodies against mouse (catalog number F-5262). As a control, they were also stained with tetramethylrhodamine isothiocyanate-conjugated antibodies against mouse (catalog number T-7657). The nuclei were stained with 
$0.1 \mu \mathrm{g} / \mathrm{ml}$ 4',6-diamidino-2-phenylindole (Polysciences, Warrington, PA, USA) in $0.9 \% \mathrm{NaCl}$. The stained embryos were visualized with a Zeiss Axiovert inverted microscope (Carl Zeiss, Oberkochen, Germany) using appropriate filters for fluorescence and differential interference contrast. For image acquisition, analog video enhancement and digital background subtraction, we used an integrated Hamamatsu CCD 2400-75i camera (Hamamatsu Photonics, Hamamatsu City, Japan), with the Hamamatsu Argus 20 image processor. Images were then transferred to a Dell GL75 computer (Dell Computers, Round Rock, TX, USA), processed using Adobe Photoshop (Adobe System Inc., San Jose, CA, USA) or the ImageJ software, based on the public domain NIH Image program (developed at the U.S. National Institutes of Health and available on the Internet at http://rsb.info.nih.gov/nih-image).

In the zebrafish embryo, new somites are formed every $20 \mathrm{~min}$ from stage 14 to $24 \mathrm{~h}$, until a total of about 30-34 somites (11). Thus, at any given time there will be somites of different ages, the older ones more anterior and the younger ones more caudal. Looking at the younger posterior somites, we can see precocious, but nevertheless perfect, striations with an anti- $\alpha$-actinin antibody (Figure 1A). The striations are in small mononucleated striated muscle precursors that can be visualized by differential interference contrast, while their connective septa are still being formed (Figure 1B,C). Other cells in the same field, visualized by the nuclear stain (Figure 1D), are not striated and are negative for $\alpha$-actinin, and therefore are either non-committed muscle precursors or other cell types.

The ability to develop tension is important for the assemblage of cytoskeletal filaments. In the in vitro model, myofibrils form from the lateral alignment of precursor structures, which were already attached and nucleated in the membrane (12). Therefore, there is an aggregation of focal adhesion struc- tures responsible for, or at least concomitant with, myofibrillogenesis. We have been studying the cytoskeletal-extracellular connection using antibodies against adhesion plaque proteins such as paxillin, talin, integrin, dystrophin (data not shown) and vinculin. We have observed a continuous adhesion structure along the connective tissue septa, which was positive for all the proteins studied since the beginning of the somitogenesis in the most caudal somites. Since a single optical plane is not enough to visualize the whole complex structure, we did a three-dimensional reconstruction of the adhesion protein structure (Figure 2A), based on images from separate focal planes (Figure 2C). Although muscle cells were quite separated from each other, there was a single continuous structure along the entire septum, and there were no separate adhesion

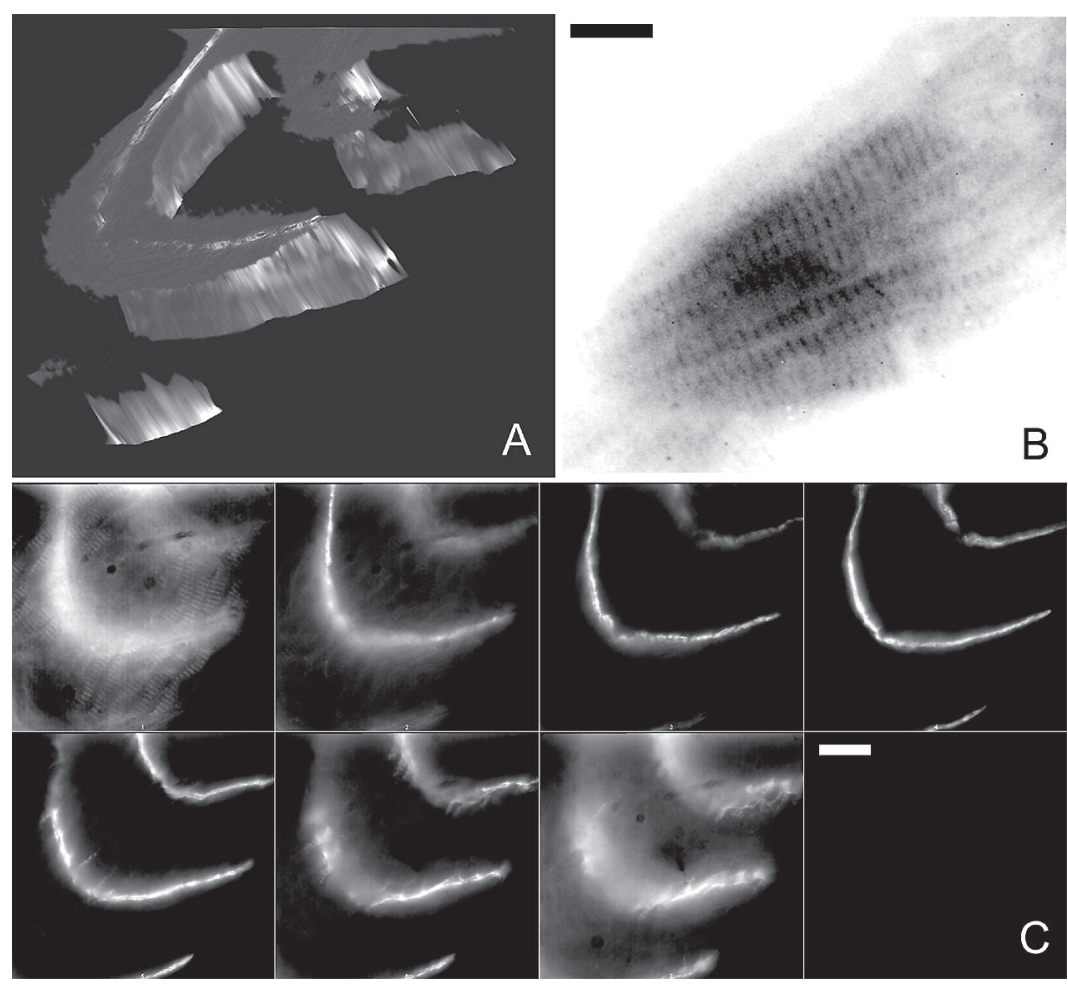

Figure 2. Somite structure. The connective tissue "box" can be seen in the three-dimensional reconstruction (A), generated from the seven separate focal planes of the immunofluorescence stain of somite 20 of zebrafish embryos with anti-vinculin antibodies (C). Between the septa, vimentin is distributed in striations in the myocytes, shown in this inverted contrast immunofluorescence stain of somite 10 of zebrafish embryos (B). Scale bars: $\mathrm{B}=10 \mu \mathrm{m} ; \mathrm{C}=20 \mu \mathrm{m}$ 
plaques.

Although the role of the intermediate filament network in myogenesis is still unclear, in our previous study we used desmin expression along development as a marker for muscle differentiation (3). We observed that the desmin expression sequence was compressed in time as compared to other models, and that its distribution in the cell was sharper and more regular than in skeletal muscle in other species. In the present study, we monitored the expression of vimentin in the zebrafish and observed that it is not down-regulated until $48 \mathrm{~h}$ after birth, and that it is perfectly striated (Figure 2B). In the usual intermediate filament protein expression model, vimentin expression is downregulated during development (13). It is not possible to use immunofluorescence to monitor vimentin expression in older animals because of their thickness and we did not study its transcriptional activity (using Northern blot for instance) in the present study.

We have previously observed significant differences between zebrafish myogenesis in situ and the conventional in vitro cell culture model (3). But if muscle differentia- tion corresponds to myofibril formation, which is based on the membrane/extracellular attachments, then much greater differences should be expected in the adhesion structures between two- and three-dimensional models. Indeed, remarkable structural and biochemical differences were observed when cells were cultured in three-dimensional collagen matrices (14). We now report that the overall distribution of the adhesion proteins in the myocytes in situ is also different from the two-dimensional in vitro distribution. We should keep in mind that muscles are exposed to several signaling molecules and positional information in situ, in addition to the three-dimensional lattice per se. We are now beginning to study more comprehensively the structural details and the protein composition of the septa, and to analyze the expression of other related proteins.

\section{Acknowledgments}

We thank R. Medeiros and V. Rodrigues for their help.

\section{References}

1. Sanger JW, Ayoob JC, Chowrashi P, Zurawski D \& Sanger JM (2000). Assembly of myofibrils in cardiac muscle cells. Advances in Experimental Medicine and Biology, 481: 89-102.

2. Ehler E, Rothen BM, Hammerle SP, Komiyama M \& Perriard JC (1999). Myofibrillogenesis in the developing chicken heart: assembly of Z-disk, M-line and the thick filaments. Journal of Cell Science, 112: 1529-1539.

3. Costa ML, Escaleira RC, Rodrigues VB, Manasfi M \& Mermelstein CS (2002). Some distinctive features of zebrafish myogenesis based on unexpected distributions of the muscle cytoskeletal proteins actin, myosin, desmin, alpha-actinin, troponin and titin. Mechanisms of Development, 116: 95-104.

4. Critchley DR (2000). Focal adhesions - the cytoskeletal connection. Current Opinion in Cell Biology, 12: 133-139.

5. Steinberg MS \& McNutt PM (1999). Cadherins and their connections: adhesion junctions have broader functions. Current Opinion in Cell Biology, 11: 554-560.

6. Culligan KG, Mackey AJ, Finn DM, Maguire PB \& Ohlendieck K (1998). Role of dystrophin isoforms and associated proteins in muscular dystrophy (Review). International Journal of Molecular Medicine, 2: 639-648.

7. Hoffman EP, Brown Jr RH \& Kunkel LM (1987). Dystrophin: the protein product of the Duchenne muscular dystrophy locus. Cell, 51 919-928.

8. Imanaka-Yoshida K, Knudsen KA \& Linask KK (1998). N-cadherin is required for the differentiation and initial myofibrillogenesis of chick cardiomyocytes. Cell Motility and the Cytoskeleton, 39: 52-62.

9. Burkin DJ \& Kaufman SJ (1999). The alpha7beta1 integrin in muscle development and disease. Cell and Tissue Research, 296: 183-190.

10. Capetanaki Y \& Milner DJ (1998). Desmin cytoskeleton in muscle integrity and function. Subcellular Biochemistry, 31: 463-495.

11. Kimmel CB, Ballard WW, Kimmel SR, Ullmann B \& Schilling TF (1995). Stages of embryonic development of the zebrafish. Developmental Dynamics, 203: 253-310.

12. Blanchard A, Ohanian V \& Critchley D (1989). The structure and function of alpha-actinin. Journal of Muscle Research and Cell Motility, 10: 280-289.

13. Kryszke $M H$, Moura-Neto V, Lilienbaum A, Paulin D \& Auclair $C$ (2001). Involvement of histone $\mathrm{H} 4$ gene transcription factor 1 in downregulation of vimentin gene expression during skeletal muscle differentiation. FEBS Letters, 491: 30-34.

14. Cukierman E, Pankov R, Stevens DR \& Yamada KM (2001). Taking cell-matrix adhesions to the third dimension. Science, 294: 17081712. 